\title{
Quantification of Dopant Distribution and the Local Band Gap in Selenium-Doped Molybdenum Disulfide
}

Andrew R. Lupini ${ }^{1}$, Wu Zhou ${ }^{1}$, Yongji Gong ${ }^{2}$, Zheng Liu ${ }^{3,4}$, Junhao Lin ${ }^{5,1}$, Sokrates T. Pantelides ${ }^{5,1}$, Stephen J. Pennycook ${ }^{6}$, Jun Lou ${ }^{3}$, Pulickel M. Ajayan ${ }^{3}$

1. Materials Science \& Technology Division, Oak Ridge National Laboratory, Oak Ridge, TN 37831 , USA

2. Department of Chemistry, Rice University, Houston, TX 77005, USA

3. Department of Mechanical Engineering \& Materials Science, Rice University, Houston, TX 77005, USA

4. School of Materials Science and Engineering, School of Electrical and Electronic Engineering Nanyang Technological University, 639798, Singapore

5. Department of Physics and Astronomy, Vanderbilt University, Nashville, TN 37235, USA

6. The University of Tennessee, Department of Materials Science and Engineering, 328 Ferris Hall, Knoxville, TN 37996, USA

Ternary two-dimensional dichalcogenide alloys exhibit compositionally modulated electronic structure. For example, we have recently shown that by substituting sulfur atoms with Se, the bandgap of $\mathrm{MoS}_{2}$ could be linearly tuned between 1.85 and $1.60 \mathrm{eV}$ as a function of Se doping level [1]. Therefore, controlled doping and quantification of the local dopant distribution becomes critical for understanding the doping behavior and its influence on the local electronic properties. However, high-accuracy and high-efficiency dopant mapping at the single atom level remains a key challenge [2], and discriminating the dopant distribution in bilayer 2D materials has not previously been achieved.

Here we describe a one-step synthesis of monolayer and bi-layer Se-doped $\mathrm{MoS}_{2}$ and demonstrate the fine-tuning of the bandgap via controlled doping [1]. Next, using atomic resolution Z-contrast imaging on an aberration-corrected scanning transmission electron microscope (STEM), we provide direct observation and quantification of individual Se dopant atoms in the $\mathrm{MoS}_{2}$ lattice, as shown in Figure 1. We develop a robust image analysis method that allows locating and quantifying the Se dopant distribution with single-atom resolution and single-layer discrimination for the first time. Furthermore, using a calibration between bandgap and doping level obtained from photoluminescence spectroscopy measurement and density- functional theory calculations, the doping level quantified via STEM imaging can be converted into bandgaps, and thus provides a measure of the local band gaps at the $10 \mathrm{~nm}$ scale.

We show that Se dopants can randomly substitute $S$ atoms in the lattice up to $75 \%$ dopant level, and there is no preference for forming any particular Se and $\mathrm{S}$ configuration in nearest neighbor chalcogen sites. Furthermore, in an AB stacking bilayer structure, we find that each layer contains similar Se doping levels, randomly distributed, suggesting a possible concurrent growth, instead of sequential growth, of bi-layer $\mathrm{MoSe}_{2(1-\mathrm{x})} \mathrm{S}_{\mathrm{x}}$ alloy under our synthesis conditions. The methodology for quantitative local band gap mapping developed in this study can also be applied to other 2D materials in general.

References:

[1] Y. Gong et al, Nano Lett., 14 (2), 442-449. (2014).

[2] D.O. Dumcenco et al., Nat. Commun. 4, 1351 (2013). 
[3] This research was supported in part by a Wigner Fellowship through the Laboratory Directed Research and Development Program of Oak Ridge National Laboratory (ORNL), managed by UTBattelle, LLC, for DOE (WZ), the DOE Basic Energy Sciences (BES), Materials Sciences and Engineering Division (ARL, SJP, STP), through a user project supported by ORNL's Center for Nanophase Materials Sciences, which is also sponsored by DOE-BES, by DOE grant DE-FG0209ER46554 (JL, STP), the Welch Foundation grant C-1716, the NSF grant DMR-0928297, the U.S. Army Research Office MURI grant W911NF-11-1-0362, the U.S. Office of Naval Research MURI grant N000014-09-1-1066, the National Research Foundation Singapore under NRF RF Award No. NRF-RF2013-08, and the Nanoelectronics Research Corporation contract S201006.
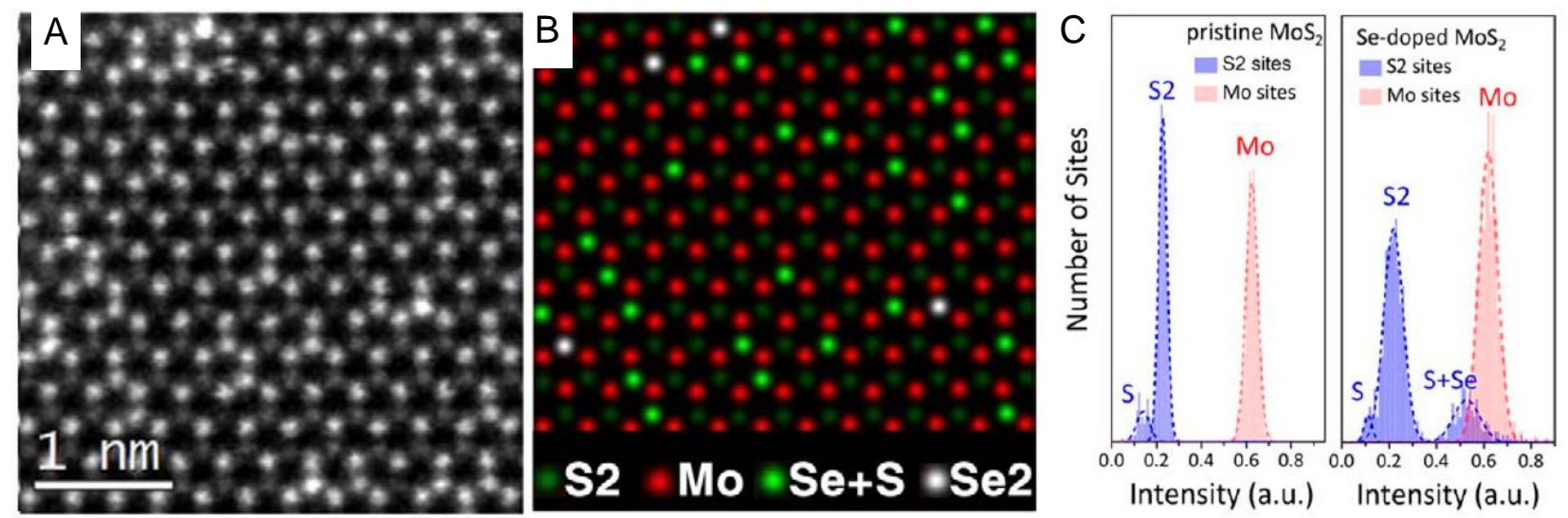

Figure 1. Atom-by-atom dopant analysis in monolayer $\mathrm{MoS}_{2}$ adapted from Ref. [1]. (A) ADF image of Se-doped MoS2. (B) Structure model obtained from histogram analysis showing the distribution of single- and double-Se substituted $\mathrm{S}_{2}$ sites. Red, Mo sites; dark green, $\mathrm{S}_{2}$ sites; bright green, $\mathrm{Se}+\mathrm{S}$ sites; white, $\mathrm{Se}_{2}$ sites. (C) Site-separated image intensity histogram analysis of pristine and Se-doped $\mathrm{MoS}_{2}$ monolayers. The dashed lines are Gaussian fits to the intensity peaks. 